\title{
Genetic Variants in the Protein S (PROS1) Gene and Protein S Deficiency in a Danish Population
}

\author{
${ }^{1}$ Department of Molecular Medicine, Aarhus University Hospital, \\ Aarhus, Denmark \\ ${ }^{2}$ Department of Clinical Medicine, Aarhus University, Aarhus, \\ Denmark \\ ${ }^{3}$ Steno Diabetes Center Aarhus, Aarhus University Hospital, Aarhus, \\ Denmark \\ ${ }^{4}$ Department of Clinical Biochemistry, Aarhus University Hospital, \\ Aarhus, Denmark
}

Ole Halfdan Larsen ${ }^{1,2}$ Alisa D. Kjaergaard ${ }^{3} \quad$ Anne-Mette Hvas 2,3 Peter H. Nissen 2,4 (D)

TH Open 2021;5:e479-e488.

\begin{abstract}
Keywords

- protein $\mathrm{S}$ deficiency

- PROS1

- venous thromboembolism

- inherited thrombophilia

Protein S (PS) deficiency is a risk factor for venous thromboembolism (VTE) and can be caused by variants of the gene encoding PS (PROS1). This study aimed to evaluate the clinical value of molecular analysis of the PROS1 gene in PS-deficient participants. We performed Sanger sequencing of the coding region of the PROS1 gene and multiplex ligation-dependent probe amplification to exclude large structural rearrangements. Free PS was measured by a particle-enhanced immunoassay, while PS activity was assessed by a clotting method.

A total of 87 PS-deficient participants and family members were included. In 22 index participants, we identified 13 PROS1 coding variants. Five variants were novel. In 21 index participants, no coding sequence variants or structural rearrangements were identified. The free PS level was lower in index participants carrying a PROS1 variant compared with index participants with no variant $(0.51$ [0.32-0.61] vs. 0.62 [0.57$\left.0.73] \times 10^{3} \mathrm{IU} / \mathrm{L} ; p<0.05\right)$. The $\mathrm{p}$.(Thr78Met) variant was associated with only slightly decreased free PS levels $\left(0.59[0.53-0.66] \times 10^{3} \mathrm{IU} / \mathrm{L}\right)$ compared with the $p$. (Glu390Lys) variant $\left(0.27[0.24-0.37] \times 10^{3} \mathrm{IU} / \mathrm{L}, p<0.01\right)$. The frequency of VTE in participants with a coding PROS1 variant was 43 and $17 \%$ in the group with normal PROS1 gene $(p=0.05)$.

In conclusion, we report 13 PROS1 coding variants including five novel variants. PS levels differ by PROS1 variant and the frequency of VTE was higher when a coding PROS1 variant was present. Hence, molecular analysis of the PROS1 gene may add clinical value in the diagnostic work-up of PS deficiency.
\end{abstract}

\begin{abstract}
Address for correspondence Peter H. Nissen, MSc, PhD, Department of Clinical Biochemistry, Aarhus University Hospital, Palle Juul-Jensens Boulevard 99, DK 8200 Aarhus, Denmark (e-mail: peteniss@rm.dk).
\end{abstract}

\section{Introduction}

Protein S (PS) is a vitamin K-dependent glycoprotein produced by the liver, which together with antithrombin and protein $\mathrm{C}$ constitutes the naturally occurring anticoagulation

received

April 23, 2021

accepted after revision

September 8, 2021
DOI https://doi.org/

$10.1055 / \mathrm{s}-0041-1736636$.

ISSN 2512-9465. factors. PS serves as a cofactor for protein C, enhancing the proteolytic activity of protein $C$. In circulation, approximately $60 \%$ of PS is bound to the complement regulatory protein C4b-binding protein, while the remaining approximately $40 \%$ is circulated as free PS contributing to anticoagulation

(c) 2021. The Author(s).

This is an open access article published by Thieme under the terms of the Creative Commons Attribution License, permitting unrestricted use, distribution, and reproduction so long as the original work is properly cited. (https://creativecommons.org/licenses/by/4.0/)

Georg Thieme Verlag KG, Rüdigerstraße 14, 70469 Stuttgart, Germany 
activity. The PS gene (PROS1) spans approximately $100 \mathrm{~kb}$ and consists of 15 translated exons that encode a 672 amino acid protein. ${ }^{1}$

Hereditary PS deficiency is an autosomal-dominant condition caused by heterozygous variants in the PROS1 gene (OMIM\#612336). ${ }^{2,3}$ Three types of PS deficiency exist: type I in which both PS level and activity are reduced. In type II PS deficiency, the circulating PS levels are normal, but the activity is reduced. Type III PS deficiency is characterized by total circulating PS in the normal range, while free PS and PS activity can be markedly reduced. ${ }^{1,4}$

Venous thromboembolism (VTE) is a multifactorial condition comprising deep venous thrombosis and pulmonary embolism. VTE occurs as a result of environmental and genetic risk factors, such as inherited thrombophilia, e.g., caused by deficiencies in the naturally occurring anticoagulants. $^{5}$ In the general population, the prevalence of PS deficiency is estimated to be 0.03 to $0.1 \%$, while the prevalence is estimated to be $2 \%$ in patients with VTE. ${ }^{6}$ Recently, the allele frequency of PROS1 variants putatively associated with PS deficiency was estimated to be $0.39 \%$ based on sequence data retrieved from the Exome Aggregation Database including more than 60,000 individual exomes. ${ }^{7}$ Clinically, PS deficiency is associated with an increased risk of $\mathrm{VTE}^{8-10}$, and an increased risk of recurrent VTE. ${ }^{8,9,11}$ However, in a recent meta-analysis, PS deficiency was shown not to be associated with recurrent VTE. ${ }^{12}$

Diagnosing hereditary PS deficiency is complicated by the fact that numerous acquired conditions can cause temporary decreases in PS levels. These include decreased synthesis of PS due to, e.g., liver disease or anticoagulant treatments using vitamin K antagonists; PS consumption by, e.g., thrombosis, surgery, and disseminated intravascular coagulation, or redistribution of complexed PS in, e.g., pregnancy, and by use of oral contraceptives. ${ }^{13}$ In addition, particularly the PS activity assays have the potential to generate false low PS values resulting in overdiagnosis of PS deficiency. ${ }^{13,14}$ Hence, molecular genetic analyses of the PROS1 gene may provide a helpful tool diagnosing hereditary PS deficiency.

The genetic spectrum of PROS1 variants includes predominantly missense variants resulting in amino acid substitutions, but also comprises nonsense variants such as small insertions and deletions, splice-site variants, and large deletions spanning one or several exons. ${ }^{8,15}$

In this study, our primary aim was to identify PROS1 variants in PS-deficient participants, while the secondary aims were to explore any possible association with PS levels as well as thrombotic phenotype based on a systematic investigation of individuals from Danish families diagnosed with PS deficiency. The overall objective was to assess the diagnostic value and clinical use of molecular genetic analysis of the PROS1 gene.

\section{Materials and Methods}

\section{Participants}

The study participants were recruited at the Thrombosis and Hemostasis Clinic at the Department of Clinical Bio- chemistry, Aarhus University Hospital. The individuals were identified by a systematic approach, using the electronic patient journal database, and it was applied to identify all patients registered with the diagnosis of PS deficiency at our department. To identify patients with contacts that predate the electronic system, the previous paper patient file system was reviewed manually. Identified PS-deficient patients were invited by letter to a new visit in the outpatient clinic and asked for potential participation in the study. In addition, newly referred participants suspected of PS deficiency in the study period were also invited to participate in the study. The ethical approval of the study did not allow identification and direct contact to the firstdegree relatives of individuals with PS deficiency. Therefore, we informed all the participants that all their first-degree relatives were welcome to participate regardless of whether they had PS deficiency or not. At our center, a thrombophilia work-up is in general performed in patients below 50 years of age due to unexplained VTE as well as unexplained cerebral arterial thrombosis event (ATE) or peripheral ATE. Furthermore, family members to individuals with severe thrombophilia, e.g., deficiency of the natural anticoagulants, undergo thrombophilia investigations. Finally, women with pregnancy complications are examined and young women are tested before use of contraceptive pills if a family disposition to venous thromboembolic disease is apparent. Hence study participants were included based on thrombophilia work-up on an index patient by one of the following causes: (1) index patient with one or more VTE events, (2) index patient with one or more ATEs, (3) index patient with pregnancy complications in one or more cases (defined as intrauterine growth restriction/birth of a smallfor-gestational-age neonate, preeclampsia, recurrent miscarriages, late pregnancy loss, or placental abruption), and (4) index patients with chance findings of low or borderline PS levels. PS deficiency was defined as two independent measurements of reduced free PS that could not be explained by temporary causes such as anticoagulants, pregnancy, or treatment with estrogens. The applied cutoff was the lower limit of the reference interval $\left(0.69 \times 10^{3}\right.$ $\mathrm{IU} / \mathrm{L})$.At inclusion, a new diagnostic thrombophilia work-up was performed including PS measurements and molecular genetic analysis of the PROS1 gene. Measurements of PS were only included if participants were either not receiving or were adequately paused in anticoagulant treatment known to affect PS levels. Furthermore, no pregnancy or treatment with estrogens was allowed for at least 3 months prior to blood sampling. Hence in some cases if the participant did not pause anticoagulant treatment at inclusion, PS data were extracted from medical records if PS values fulfilling the requirements were available within 1 year from the inclusion date.

Information on thromboembolic events and pregnancy complications was recorded based on systematic interview of the participants supported by medical records.

Eventually, we included 87 participants of which 70 had PS deficiency while five participants were included with inconclusive PS status. When possible, PS measurements 
fulfilling the requirements (see above) were obtained and evaluated in a total of 55 participants. Study participants were included from November 2015 to September 2018.

\section{Protein S Measurements}

Free PS was measured on the Sysmex CS2100i coagulation system by a particle-enhanced immunoassay using the commercial INNOVANCE Free PS Ag kit (Siemens Healthineers, Erlangen, Germany). The reference interval was 0.69$1.37 \times 10^{3} \mathrm{IU} / \mathrm{L}$ (locally determined $95 \%$ reference interval based on 105 blood donors). PS activity was measured on the ACL TOP 550 system by a clotting method using the commercial Hemosil Protein S Activity kit (Instrumentation Laboratory, Munich, Germany). The reference interval was 0.75$1.40 \times 10^{3} \mathrm{IU} / \mathrm{L}$ (locally determined $95 \%$ reference interval based on 50 blood donors).

\section{Genetic Analysis}

\section{Polymerase Chain Reaction and Sequencing}

DNA isolation was performed by use of either the QiaSymphony DSP mini kit (Qiagen, Hilden, Germany) or the Maxwell 16 Blood DNA Purification Kit (Promega, Nacka, Sweden) according to the manufacturer's instructions.

Protein coding exons and flanking intronic regions of the PROS1 gene were amplified using previously reported primers. ${ }^{15}$ The primers were modified with M13 linkers to facilitate sequencing. The polymerase chain reaction (PCR) products were purified by use of exonuclease and shrimp alkaline phosphatase digestion (ExoSAP-IT) as recommended by the manufacturer (Life Technologies Europe BV, Roskilde, Denmark). The purified fragments were bidirectionally sequenced using M13 sequencing primers (M13F: 5' - GTAAAACGACGGCCAG - 3' and M13R: 5' CAGGAAACAGCTATGAC $-3^{\prime}$ ) and BigDye terminator version 1.1 (Life Technologies). The sequencing reactions were ethanol-precipitated and separated on an Applied Biosystems 3500 or $3500 x$ l Genetic Analyzer (Life Technologies). Sequence traces were aligned to NM_000313 (PROS1) by use of SeqScape software (version 2.7, Life Technologies).

Nomenclature of variants follows current guidelines. ${ }^{16}$

\section{Multiplex Ligation-Dependent Probe Amplification}

Index participants were analyzed for large structural rearrangements, using multiplex ligation-dependent probe amplification (MLPA). We used the SALSA MLPA probemix P112A3 PROS1 (MRC-Holland, Amsterdam, The Netherlands). Using this procedure, all PROS1 exons, except exon 2 and exon 15 , were targeted.

MLPA was performed essentially as described by the manufacturer. In brief, genomic DNA was denatured at $98^{\circ}$ $\mathrm{C}$ for 5 minutes and hybridization of probes was performed by incubating each sample with the probe mix at $60^{\circ} \mathrm{C}$ for 16 hours. Following this, ligation proceeded at $54^{\circ} \mathrm{C}$ for 15 minutes. The resulting DNA with hybridized and ligated probes was amplified using the PCR primers supplied. The amplified fragments were separated on an Applied Biosystems 3500 or $3500 x l$ Genetic Analyzer (Life Technologies) including the GS500ROX size standard (Life Technologies). The GeneMapper Software (version 4.1, Life Technologies) was used for visualization of fragment analysis data. MLPA profiles were assessed by visual inspection of the peaks.

\section{Bioinformatics Analysis}

We performed in silico assessment of missense variants using the prediction tools PolyPhen2, SIFT, and MutationTaster2. ${ }^{17-19}$ Two intronic variants were assessed for possible activation of cryptic splice sites using the Splice Site Prediction tool ${ }^{20}$ and the NetGene2 Server. ${ }^{21}$ A putative promoter variant was assessed for possible disruption of promoter binding sites by the Neural Network Promoter Prediction tool. ${ }^{22}$

Further, ClinVar (https://www.ncbi.nlm.nih.gov/clinvar/), dbSNP (https://www.ncbi.nlm.nih.gov/snp/), and gnomAD (https://gnomad.broadinstitute.org/) were assessed for information on each specific variant.

\section{Classification of Variants}

The pathogenicity of the variants was evaluated using the classification system jointly proposed by the American College of Medical Genetics and Genomics and the Association for Molecular Pathology (ACMG-AMP). ${ }^{23}$ This system classifies sequence variants in five classes as either pathogenic (class 5 ), likely pathogenic (class 4 ), uncertain significance (VUS, class 3 ), likely benign (class 2 ), or benign (class 1 ). The classification is based on a range of criteria, including the nature of the variant (e.g., nonsense or amino acid substitution), outcome of in silico predictions, segregation of the variant, and several other criteria. Assessment of each of these elements resulted in the assignment of a criterion in case the condition was fulfilled. All assigned criteria for each variant were evaluated using the ACMG-AMP as reference. All assigned criteria are provided in -Supplementary Table S1 (online only).

\section{Statistics}

The majority of the quantitative data did not follow a Gaussian distribution; thus all data were expressed as median with corresponding interquartile range (i.e., 25th and 75th percentiles) except for age where median with range was applied. Pairwise comparisons between two groups were performed using the Mann-Whitney test. For categorical data, Fisher's exact test was used. A probability $(p)$ of 0.05 was used as the threshold for statistical significance.

Statistical analyses and figures were performed using GraphPad Prism version 8 (GraphPad Software, Inc., La Jolla, California, United States).

\section{Results}

\section{Clinical Characteristics}

Characteristics of the study population are shown in -Table 1. We included 75 participants of which 70 had PS deficiency while five participants were included with inconclusive PS status (borderline free PS results or contradictory PS measurements). Of these, 43 were index 
e482 Genetic Variants in the Protein S (PROS1) Gene Larsen et al.

Table 1 Characteristics of the study population

\begin{tabular}{|l|l|l|l|}
\hline & Index participants & PS-deficient relatives & Healthy relatives \\
\hline Participants, $N$ & 43 & 32 & 12 \\
\hline Women, $N(\%)$ & $34(79)$ & $21(66)$ & $7(58)$ \\
\hline Age at inclusion \pm SD, y & $45 \pm 17$ & $45 \pm 16$ & $51 \pm 12$ \\
\hline ACT, $N(\%)$ & $22(51)$ & $8(25)$ & $0(0)$ \\
\hline ACT in high-risk situations, $N(\%)$ & $18(42)$ & $16(50)$ & $0(0)$ \\
\hline No ACT, $N(\%)$ & $3(7)$ & $8(25)$ & $12(100)$ \\
\hline
\end{tabular}

Abbreviation: ACT, anticoagulant treatment; PS, protein S; SD, standard deviation.

Note: Healthy relatives: relatives with normal free protein $S$ levels.

participants, presenting with VTE, ATE, pregnancy complications, or reduced PS levels, and 32 were PS-deficient relatives. In addition, 12 individuals, recruited as part of cascade screening in families with PS deficiency, had normal free PS levels and normal PROS1 genotype.

\section{PROS1 Variants and Classification}

By a Sanger sequencing approach, we identified 16 different PROS1 variants ( - Table 2 ). Three variants were located in noncoding regions ( $5^{\prime}$ untranslated region [5'UTR] and exon flanking intronic regions). Ten were missense variants resulting in amino acid substitutions, while three were nonsense variants, either as a result of nucleotide substitutions resulting in a premature stop codon $(N=2)$ or due to a $1 \mathrm{bp}$ deletion resulting in a frameshift and a premature stop codon $(N=1)$. Four variants were not previously reported but were present in dbSNP. Five variants were novel and neither published nor present in dbSNP, ClinVar, or gnomAD, while seven variants were previously published.

Using the ACMG-AMP criteria for variant classification, ${ }^{23}$ three variants were classified as likely pathogenic (class 4 ), while the three nonsense variants were classified as pathogenic (class 5). We classified seven of the variants as VUS (class 3). This classification is due to several factors, e.g., lack of family members to follow segregation of the variant with PS deficiency and disagreement of functional consequence between in silico prediction methods. The three noncoding variants were classified as class 2 , likely benign variants (-Supplementary Table S1 [online only]).

To identify potential large complex rearrangements of the PROS1 gene, such as deletions spanning one or more exons, MLPA was performed in all index participants (two samples failed due to technical reasons). We did not identify large

Table 2 Variants of the PROS1 gene identified in Danish participants

\begin{tabular}{|c|c|c|c|c|c|}
\hline PROS1 region & Nucleotide change & $\begin{array}{l}\text { Predicted amino } \\
\text { acid change }\end{array}$ & $\begin{array}{l}\text { dbSNP reference } \\
\text { number }\end{array}$ & ACMG-AMP classification & References \\
\hline Exon 1 (5'UTR) & c. $-43 G>A$ & - & rs370938580 & Likely benign & Not reported \\
\hline Exon 1 & c. $32 \mathrm{~T}>\mathrm{C}$ & p.(Leu11Pro) & NA & Uncertain significance & Novel \\
\hline Intron 1 & c. $77-32 A>G$ & - & rs778070336 & Likely benign & Not reported \\
\hline Exon 2 & c. $200 \mathrm{~A}>\mathrm{C}$ & p.(Glu67Ala) & rs766423432 & Uncertain significance & Not reported \\
\hline Exon 2 & c. $233 C>T$ & p.(Thr78Met) & rs6122 & Likely pathogenic & $25,32-34$ \\
\hline Exon 7 & c. $698 \mathrm{G}>\mathrm{A}$ & p.(Arg233Lys) & rs41267007 & Uncertain significance & 35 \\
\hline Intron 8 & c. $728-20 G>A$ & - & rs78230833 (G>A) & Likely benign & 36 \\
\hline Exon 9 & c.913C > T & p. $\left(G \ln 305^{*}\right)$ & rs1395378093 & Pathogenic & 33 \\
\hline Exon 10 & c.992C > T & p.(Thr331lle) & NA & Uncertain significance & Novel \\
\hline Exon 10 & c. $1153 A>G$ & p.(Met385Val) & rs766423432 & Uncertain significance & Not reported \\
\hline Exon 11 & c. $1168 G>A$ & p.(Glu390Lys) & NA & Likely pathogenic & 37,38 \\
\hline Exon 11 & c. $1241 \mathrm{~T}>\mathrm{C}$ & p.(Phe414Ser) & NA & Uncertain significance & Novel \\
\hline Exon 12 & c. $1351 \mathrm{C}>\mathrm{T}$ & p. $\left(\operatorname{Arg} 451^{*}\right)$ & rs5017717 & Pathogenic & 33,37 \\
\hline Exon 12 & c.1468del & p.(Ile490Leufs*6) & NA & Pathogenic & Novel \\
\hline Exon 13 & c. $1501 \mathrm{~T}>\mathrm{C}$ & p.(Ser501Pro) & rs121918472 & Uncertain significance & 37,39 \\
\hline Exon 13 & c. $1577 \mathrm{~T}>\mathrm{C}$ & p.(Leu526Ser) & NA & Likely pathogenic & Novel \\
\hline
\end{tabular}

Abbreviation: dbSNP, Single Nucleotide Polymorphism Database. 


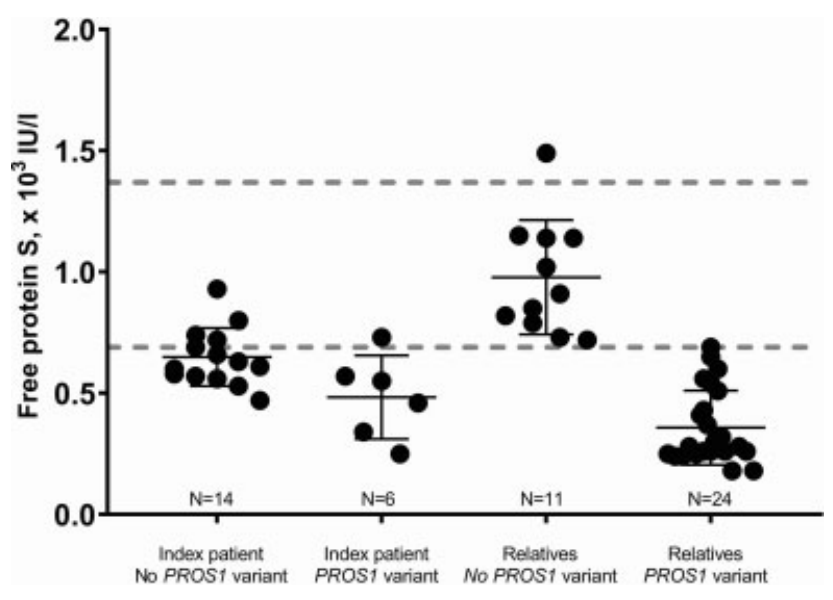

Fig. 1 Free protein $S$ levels in protein S-deficient index participants with a PROS1 variant or with normal PROS1 sequence and relatives with PROS1 variant or normal PROS1 sequence. PROS1 variants included were: p.(Glu67Ala), p.(Thr78Met), p.(Gln305*), p.(Thr331lle), p. (Met385Val), p.(Glu390Lys), p.(Arg451*), p.(Ile490Leufs*6), p. (Ser501Pro), p.(Leu526Ser). The reference interval for free protein $S$ $\left(0.69-1.37 \times 10^{3} \mathrm{IU} / \mathrm{L}\right)$ is indicated by dotted horizontal lines.

rearrangements of the PROS1 gene in any participants in this study.

\section{Protein S Levels Differ by PROS1 Variant}

We evaluated PS levels, and found that the free PS level was lower in index participants carrying a coding PROS1 variant compared with index participants with no PROS1 variant $\left(0.51[0.32-0.61] \times 10^{3} \mathrm{IU} / \mathrm{L}(N=6)\right.$ vs. $0.62[0.57-0.73] \times 10^{3}$ $\mathrm{IU} / \mathrm{L}(N=14) ; p=0.03 ;$ - Fig. 1$)$. One exception was a participant double heterozygous for the p.(Thr78Met) and the $\mathrm{p}$. (Arg233Lys) variants presenting with a free PS of 0.73 (-Fig. 1). As expected, the control group consisting of individuals included as part of family screening, with no PROS1 variants, had free PS levels in the normal range ( $N=11$, data from one individual missing, - Fig. 1 ).

In the available cases, there were no difference between free PS and PS activity for participants with no PROS1 variant (0.62 [0.57-0.73] $\times 10^{3} \quad \mathrm{IU} / \mathrm{L} \quad(N=14)$ vs. (0.65 [0.51$\left.0.76] \times 10^{3} \mathrm{IU} / \mathrm{L}(N=10), p=0.99\right)$. Likewise, no difference was observed in free PS and PS activity for participants with a PROS1 variant $\left(0.51[0.32-0.61] \times 10^{3} \mathrm{IU} / \mathrm{L}(N=6)\right.$ vs. $(0.51$ $\left.[0.38-0.62] \times 10^{3} \mathrm{IU} / \mathrm{L}(N=3), p=0.90\right)$.

To explore genotype-phenotype correlations, free PS levels were grouped by variant in cases where data on two or more individuals were available. - Fig. 2 indicates that the p.(Thr78Met) variant resulted in only slightly decreased free PS levels (0.59 [0.53-0.66] × $\left.10^{3} \mathrm{IU} / \mathrm{L}, N=6\right)$ compared with, e.g., the p.(Glu390Lys) variant $\left(0.27[0.24-0.37] \times 10^{3} \mathrm{IU} / \mathrm{L}\right.$, $N=7, p=0.001)$. One participant included during family screening and carrying the p.(Thr78Met) had free PS level of $0.69 \times 10^{3} \mathrm{IU} / \mathrm{L}$ corresponding to the lower reference limit.

To further evaluate if the reduced free PS levels, associated to the p.(Thr78Met) variant, might be due to interference in the free PS assay, rather than representing a quantitative defect, we compared the free PS and PS activity levels for this variant (-Fig. 3). The median level of the PS activity meas-

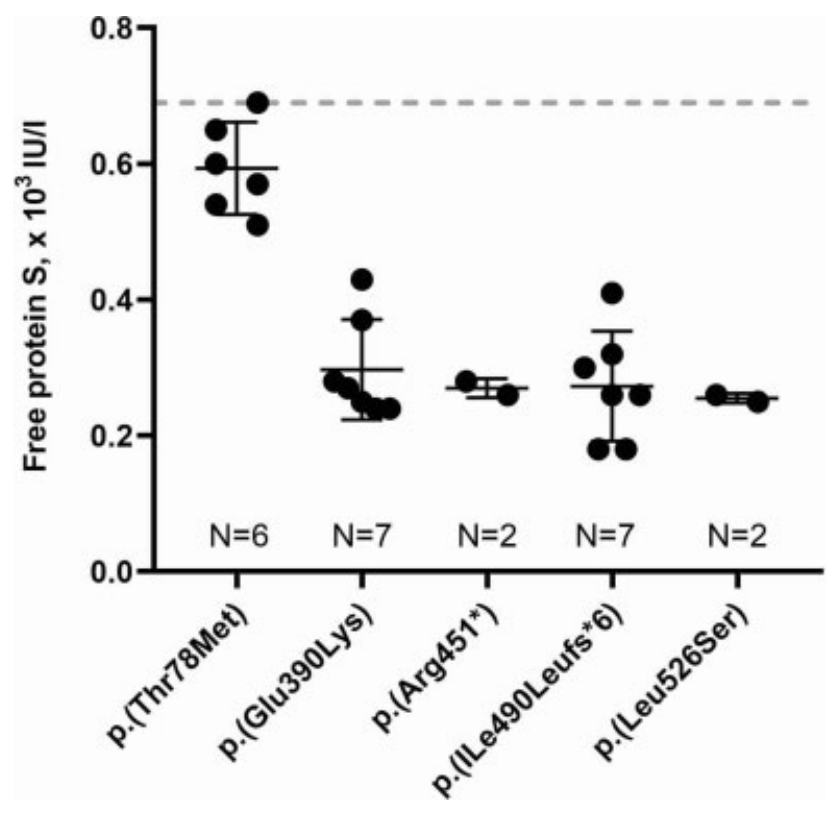

Fig. 2 Free protein S levels in protein S-deficient participants carrying five specific PROS1 variants. The lower reference limit for free protein $S$ $\left(0.69 \times 10^{3} \mathrm{IU} / \mathrm{L}\right)$ is indicated by a dotted horizontal line.

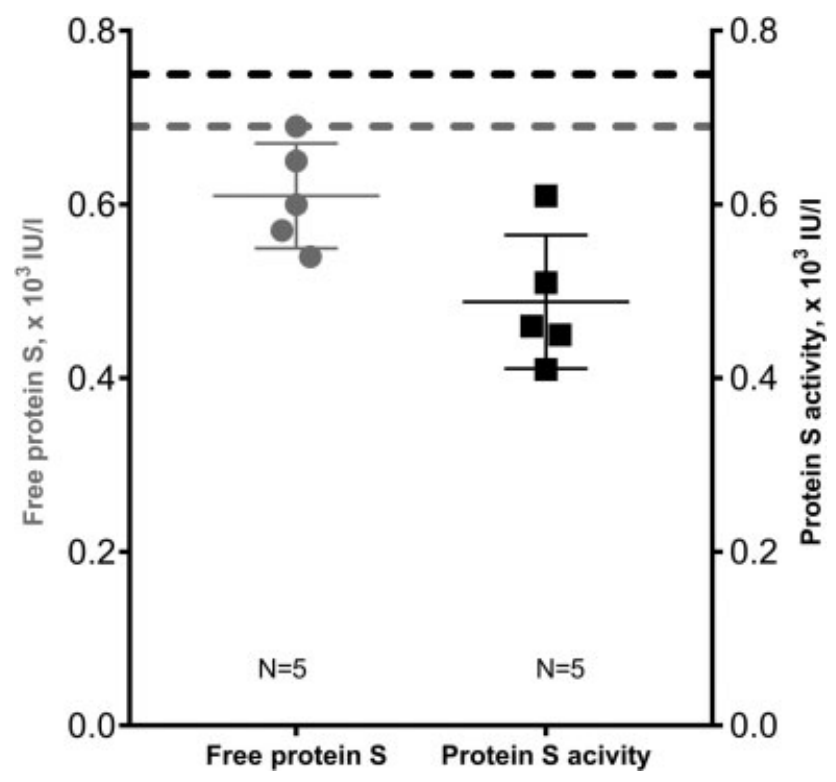

Fig. 3 Free protein $\mathrm{S}$ and protein $\mathrm{S}$ activity for participants heterozygous for the p.(Thr78Met) variant of the PROS1 gene. Gray dots represent participants with free protein $S$ values $(N=5)$. The gray broken line indicates the lower reference limit $\left(0.69 \times 10^{3} \mathrm{IU} / \mathrm{L}\right)$ of the free protein $S$ assay. Protein $S$ activity measurements were available for the same five, presented as black squares with the corresponding lower reference limit of $0.75 \times 10^{3} \mathrm{IU} / \mathrm{L}$ in the black broken line.

urements was $0.46[0.43-0.56] \times 10^{3} \mathrm{IU} / \mathrm{L}(N=5$, reference range: $\left.0.69-1.37 \times 10^{3} \mathrm{IU} / \mathrm{L}\right)$ compared with 0.60 [0.56$0.69] \times 10^{3} \mathrm{IU} / \mathrm{L}\left(N=5\right.$, reference range: $0.75-1.40 \times 10^{3}$ $\mathrm{IU} / \mathrm{L})$ for the free PS assay $(p=0.06)$.

\section{Reason for Referral and PROS1 Variant Detection}

We identified a total of 23 coding variants in 43 index participants, resulting in a crude detection rate of $53 \%$. 
Table 3 Clinical characteristics of 75 study participants (43 index participants and 32 family members)

\begin{tabular}{|c|c|c|c|c|c|c|c|c|}
\hline $\begin{array}{l}\text { Nucleotide } \\
\text { change }\end{array}$ & $\begin{array}{l}\text { Predicted } \\
\text { amino } \\
\text { acid change }\end{array}$ & $\begin{array}{l}\text { Participants, } \\
N \text { (families) }\end{array}$ & $\begin{array}{l}\text { VTE, } \\
N(\%)\end{array}$ & $\begin{array}{l}\text { Recurrent } \\
\text { VTE, } \\
N(\%)\end{array}$ & $\begin{array}{l}\text { Index } \\
\text { participants } \\
\text { with family } \\
\text { history } \\
\text { of VTE, } \\
N(\%)\end{array}$ & $\begin{array}{l}\text { ATE, } \\
N(\%)\end{array}$ & $\begin{array}{l}\text { Recurrent } \\
\text { ATE, } N(\%)\end{array}$ & $\begin{array}{l}\text { Pregnancy } \\
\text { complications, } \\
N \text { (\% of women) }\end{array}$ \\
\hline $\begin{array}{l}\text { No variant } \\
\text { detected }\end{array}$ & - & $18(18)$ & $3(17)$ & $1(6)$ & $4(22)$ & $7(39)$ & $1(6)$ & $6(33)$ \\
\hline c. $-43 G>A$ & - & $2(1)$ & $0(0)$ & $0(0)$ & $1(100)$ & $0(0)$ & $0(0)$ & $0(0)$ \\
\hline c. $32 \mathrm{~T}>\mathrm{C}$ & p.(Leu11Pro) & $1(1)$ & $1(100)$ & $1(100)$ & $1(100)$ & $0(0)$ & $0(0)$ & $0(0)$ \\
\hline c. $77-32 A>C$ & - & $1(1)$ & $1(100)$ & $0(0)$ & $0(0)$ & $0(0)$ & $0(0)$ & $0(0)$ \\
\hline c. $200 \mathrm{~A}>\mathrm{C}$ & p.(Glu67Ala) & $2(2)$ & $2(100)$ & $0(0)$ & $1(50)$ & $0(0)$ & $0(0)$ & $0(0)$ \\
\hline c. $233 C>T$ & p.(Thr78Met) & $9(3)$ & $0(0)$ & $0(0)$ & $2(22)$ & $2(22)$ & $0(0)$ & $1(13)$ \\
\hline $\begin{array}{l}\text { c. } 233 \mathrm{C}>\mathrm{T} ; \\
\text { c. } 698 \mathrm{C}>\mathrm{A}\end{array}$ & $\begin{array}{l}\text { p.(Thr78Met); } \\
\text { p.(Arg233Lys) }\end{array}$ & $1(1)$ & $1(100)$ & $0(0)$ & $1(100)$ & $0(0)$ & $0(0)$ & $0(0)$ \\
\hline c. $728-20 G>A$ & - & $1(1)$ & $0(0)$ & $0(0)$ & $1(100)$ & $1(100)$ & $0(0)$ & $0(0)$ \\
\hline c.913C > T & p. $\left(G \ln 305^{*}\right)$ & $1(1)$ & $0(0)$ & $0(0)$ & $1(100)$ & $0(0)$ & $0(0)$ & $0(0)$ \\
\hline c.992C $>$ T & p.(Thr331lle) & $1(1)$ & $0(0)$ & $0(0)$ & $1(100)$ & $0(0)$ & $0(0)$ & $0(0)$ \\
\hline$c .1153 \mathrm{~A}>\mathrm{G}$ & p.(Met385Val) & $1(1)$ & $0(0)$ & $0(0)$ & $1(100)$ & $0(0)$ & $0(0)$ & $1(100)$ \\
\hline c. $1168 \mathrm{G}>\mathrm{A}$ & p.(Glu390Lys) & $15(6)$ & $9(56)$ & $5(31)$ & $5(83)$ & $1(6)$ & $0(0)$ & $1(13)$ \\
\hline c. $1241 \mathrm{~T}>\mathrm{C}$ & p.(Phe414Ser) & $1(1)$ & $1(100)$ & $0(0)$ & $0(0)$ & $0(0)$ & $0(0)$ & $0(0)$ \\
\hline c. $1351 C>T$ & p. $\left(\operatorname{Arg} 451^{*}\right)$ & $3(1)$ & $1(33)$ & $1(33)$ & $0(0)$ & $1(33)$ & $1(33)$ & $0(0)$ \\
\hline c.1468del & p.(Ile490Leufs*6) & $12(1)$ & $5(42)$ & $4(33)$ & $1(100)$ & $1(8)$ & $0(0)$ & $0(0)$ \\
\hline c. $1501 \mathrm{~T}>\mathrm{C}$ & p.(Ser501Pro) & $2(2)$ & $2(100)$ & $1(50)$ & $0(0)$ & $0(0)$ & $0(0)$ & $0(0)$ \\
\hline c. $1577 \mathrm{~T}>\mathrm{C}$ & p.(Leu526Ser) & $4(1)$ & $2(50)$ & $0(0)$ & $1(100)$ & $0(0)$ & $0(0)$ & $2(100)$ \\
\hline
\end{tabular}

Abbreviations: ATE, arterial thrombosis event; VTE, venous thromboembolism.

To investigate the detection rate of the molecular genetic analysis in a clinical setting, different scenarios were established based on the reason for referral. Hence, we analyzed the detection rate in the subsets of index participants where the reason for referral was one of the three major indications: (1) a personal history of VTE $(N=18)$, (2) ATE $(N=9)$, or (3) pregnancy complications $(N=4)$.

In $83 \%$ ( 15 of 18 ) of the index participants referred due to VTE, a coding PROS1 variant was identified. For index participants referred due to ATE the detection rate was $11 \%$ ( 1 of 9 ). When the reason for referral was pregnancy complications, the detection rate was $25 \%$ ( 1 of 4 ).

\section{PROS1 Variants and Venous Thromboembolism}

The clinical characteristics of PS-deficient participants are summarized in -Tables 3 and $\mathbf{4}$. Of 18 index participants with no detectable PROS1 variants, three participants experienced VTE (17\%). Of these, one participant had two events at the age of 15 and 24 years. A total of 54 participants carried a coding PROS1 variant. Of these, $43 \%$ experienced one or more VTEs. Testing the hypothesis that VTE is independent of having a variant in the PROS1 gene was just rejected by Fisher's exact test at the $5 \%$ significance level $(p=0.05)$. Thus, our data cannot rule out, that the apparent overweight of participants with VTE, which were heterozygous for a PROS1 variant, was a chance finding.

\section{Arterial Thromboembolism and Pregnancy Complications}

Arterial thromboembolism was more frequent in the group of participants with normal PROS1 gene compared with participants with a PROS1 variant (39 vs. $11 \%, p=0.01$ ). Recurrent ATE was reported in one case in both groups of $\sim$ Table 4 .

A range of pregnancy complications was reported: abruptio placentae, preeclampsia, early abortion, spontaneous abortion, intrauterine fetal death, and premature birth. Pregnancy complications were reported more frequent in the group of participants with normal PROS1 gene compared with the group with a PROS1 variant (33 vs. 9\%, $p=0.02$, - Table 4). However, recurrent pregnancy complications were reported in four of the five cases in the group of PROS1 variant carriers and in two of six cases in the group with normal PROS1 gene (-Table 4).

\section{Discussion}

Since the heritable nature of PS deficiency was first acknowledged as a contributing factor to the development of VTE, ${ }^{2,3}$ 
Table 4 Thromboembolic events and pregnancy by variant status (PROS1 variants class 3-5)

\begin{tabular}{|l|l|l|l|}
\hline & PROS1 coding variant & Normal PROS1 gene & $p$-Value \\
\hline Total number of participants & 54 & 18 & \\
\hline Women & $35(65)$ & $18(100)$ & 0.32 \\
\hline Age at inclusion in years & $45(19-80)$ & $35(20-60)$ & 0.02 \\
\hline Age at first venous thromboembolic event & $30(14-64)$ & $21(15-27)$ & 0.09 \\
\hline Venous thromboembolic events & $23(43)$ & $3(17)$ & 0.05 \\
\hline Deep vein thrombosis & $12(52)$ & $3(100)$ & \\
\hline Pulmonary embolism & $4(17)$ & $0(0)$ & \\
\hline Deep vein thrombosis and pulmonary embolism & $5(22)$ & $0(0)$ & \\
\hline Other (vena porta or retinal vein thrombosis) & $2(9)$ & $0(0)$ & \\
\hline Recurrent venous thromboembolic events & $12(52)$ & $1(33)$ & 0.01 \\
\hline Arterial thromboembolism & $6(11)$ & $7(39)$ & \\
\hline Recurrent arterial thromboembolic events & $1(2)$ & $1(6)$ & 0.02 \\
\hline Pregnancy complications & $5(9)$ & $6(33)$ & \\
\hline Recurrent pregnancy complications & $4(7)$ & $2(11)$ & \\
\hline
\end{tabular}

Note: Values are $N(\%)$ or median (range).

considerable progress has been made in understanding the contribution of genetic variants of the PROS1 gene to the disease outcomes of PS deficiency. However, it is unclear whether molecular genetic analysis of the PROS1 gene provides further clinical insights into the diagnostic work-up of PS deficiency, and in a recent guideline, the clinical value of genetic analysis of PROS1 is not directly addressed. ${ }^{14}$

In this study, we performed molecular genetic analysis of participants with PS deficiency and identified 16 different PROS1 variants of which nine were not previously reported as variants associated with PS deficiency. These results expand the spectrum of PROS1 variants associated with PS deficiency and support other studies showing heterogeneity in the genetic background of PS deficiency. ${ }^{15,24,25}$

We classified the variants using the ACMG-AMP criteria. ${ }^{23}$ Seven variants were classified as VUS making these a challenge in terms of clinical action. In the present study two factors were predominantly decisive in a classification as a VUS: disagreement in the consequence of the variant predicted by in silico prediction tools and lack of information on segregation of the variant. The first is a well-known problem in prediction tools, each tool having different ability to correctly assess the functional consequence of the variants. In the ACMG-AMP criteria, multiple lines of computational evidence supporting a deleterious effect of a variant weigh as supporting evidence of pathogenicity. However, as reviewed by Masica and Karchin, PolyPhen2 and SIFT, two of the prediction tools used in the present study, have a prediction accuracy ranging from 62 to $80 \%$ dependent on the dataset assessed. ${ }^{26}$ As a consequence, the ACMG-AMP classifier related to computational evidence may often be the decisive classification element tipping a variant from likely pathogenic to a VUS.

Large deletions of the PROS1 gene have been associated with PS deficiency in individuals where no other PROS1 variants were identified; however, the extent of this type of variant seems to vary a great deal. Caspers and coauthors identified a large deletion in five of 185 individuals, ${ }^{15}$ while a smaller study identified a PROS1 deletion in 33\% of PSdeficient probands. ${ }^{27}$ In the present study, we did not identify large complex rearrangements of the PROS1 gene by use of MLPA.

The c.-43G $>$ A variant, located in the $5^{\prime} \mathrm{UTR}$, was of interest since a recent study identified a c.-39C $>$ T variant that was shown in vitro to introduce a new translation initiation codon, and consequently a premature stop codon and suggested to cause PS deficiency. ${ }^{28}$ However, in the present study, one family member heterozygous for the c.$43 \mathrm{G}>\mathrm{A}$ variant had normal PS levels, indicating that this is a variant with no impact on the PS phenotype.

We observed statistically significantly lower PS levels in index participants heterozygous for a PROS1 variant than in index participants with normal PROS1 gene. In addition, our data suggest that protein coding variants are associated with variable phenotypes of PS plasma levels, particularly driven by the observations on the p.(Thr78Met) variant. Participants heterozygous for the $\mathrm{p}$.(Thr78Met) variant had higher free PS levels than participants heterozygous for, e.g., the p. (Glu390Lys) variant ( $p=0.001,-$ Fig. 2 ). To rule out that the p.(Thr78Met) variant was not a normal variant interfering with the free PS assay, resulting in false low free PS values, we compared free PS results to PS activity in available cases with both measurements. There was no statistically significant difference between PS activity values and free PS values, indicating that the p.(Thr78Met) is a variant that causes only slightly reduced PS levels. Further, none of the nine participants heterozygous for the $\mathrm{p}$.(Thr78Met) variant presented with VTE. However, two presented with ATE and one experienced pregnancy complications. To our knowledge, this is the first study to show genotype-phenotype correlation on the variant level. 
The p.(Glu390Lys) was the most frequent variant identified in $26 \%$ of the index participants in the present study group. In an early study of Danish PS-deficient families, it was suggested based on microsatellite and haplotype analysis that the p.(Glu390Lys) variant was a founder variant. This is consistent with the high frequency of this variant among PS-deficient index participants in our study. If this is also the case for the $\mathrm{p}$.(Thr78Met) variant, which was present in $17 \%$ of the index participants, remains to be investigated.

Apart from several case reports, a few studies of cohorts of similar size to the present study have reported on the association between PROS1 variants and clinical manifestations such as VTE. ${ }^{24,25}$ We observed a higher frequency (43\%) of PS-deficient participants with VTE carrying a PROS1 variant than in the group not carrying a PROS1 variant (17\%); however, the data were not statistically significant $(p=0.05)$. In a study of cases of unprovoked idiopathic fatal pulmonary embolism, it was shown by a whole exome sequencing approach that the risk of death was highly increased in cases heterozygous for a PROS1 variant compared with the control group (odds ratio $=56.4, p=0.001$ ). ${ }^{29}$

In our cohort the clinical data on arterial thromboembolic events and pregnancy complications suggest an opposite dependency compared with VTE. For both ATE and pregnancy complications, the frequency of events was higher in the groups with no PROS1 variant compared with PS-deficient participants heterozygous for a PROS1 variant $(p=0.02)$. However, recurrent pregnancy complications were more frequent in the group of PROS1 gene variant carriers.

In the present study, the crude detection rate of a coding PROS1 variant was $52 \%$, while the detection rate increased substantially to $83 \%$ for index participants referred due to VTE. Caspers and colleagues showed a variant detection rate of $43 \%$ with an inclusion criterion of participants with a personal history of a thromboembolic event in association with reduced PS activity levels. ${ }^{15}$ In the opposite end of the variant detection spectrum, the study by Ten Kate and colleagues identified a PROS1 variant in 35 of 36 PS-deficient probands, corresponding to a detection rate of $97 \%{ }^{30}$ Contrary to this, we observed low detection rates of 11 and 25\% when the reason for referral was ATE and pregnancy complications, respectively. The large spectrum of variant detection rates and our results show that the establishment of PS deficiency and a personal history of VTE increase the likelihood of identifying a PROS1 variant.

These findings together, bearing the challenges in the plasma assays for PS in mind, suggest that PROS1 genotyping may be a useful tool in the diagnostic work-up of PS deficiency particularly for VTE. When the reason for referral is ATE and pregnancy complications, the value of molecular genetic analysis of the PROS1 gene is more questionable.

Further, our study suggests that it may be possible to stratify treatment based on genotype, since carriers of the $p$. (Thr78Met) variant seem to be less prone to VTE than carriers of, e.g., the p.(Glu390Lys) variant. However, this strategy needs to be confirmed in future clinical studies, and the present study does not allow us to conclude causal effects.
The present study represents the largest cohort of Danish PS-deficient participants thoroughly studied by molecular genetic analysis. Only few studies have reported systematically on the association between PROS1 genotype and thromboembolic events. Thus, our data represent a step forward and an opportunity to improve the diagnostic work-up of patients with PS deficiency. Some limitations of the study need consideration.

As the study participants were recruited based on medical records, selection bias must be considered as index participants were included based not only on decreased PS levels but also on thrombotic events. This makes it possible that decreased PS levels may be chance findings with no causal effect. Further, it was not possible to perform follow-up, thus we cannot reject that some study participants have developed events later in life. We measured free PS as standard care and PS activity in several cases. However, total PS was not measured in this study, limiting the ability to distinguish between type I and type III PS deficiency. The use of total PS measurements is not common practice as it will not contribute significantly to the final diagnosis, ${ }^{13}$ and distinguishing between type I and type III PS deficiency will rarely impact the treatment or counseling of the patient and family. In our study, all the 18 participants without a PROS1 variant were women. Previous studies have indicated that the PS levels are lower in women than in men. ${ }^{31}$ Therefore, it is possible that these women may not have genetically determined PS deficiency, which is supported by the genetic data and lack of PROS1 variants. The association of thromboembolic events and PROS1 variant relies on small groups. Increasing the number of participants might have revealed more reliable associations. Finally, the evaluation of the variants could have been improved by performing functional in vitro studies of novel variants. To compensate for this limitation, we performed a stringent classification based on current ACMG guidelines.

In conclusion, this study expands the spectrum of genetic variants in the PROS1 gene associated with PS deficiency and reports a genotype-phenotype correlation on the variant level. A total of 16 different PROS1 variants were detected, of which 13 were classified as pathogenic, likely pathogenic, or VUS. We observed that the p.(Thr78Met) variant in the present dataset was associated with higher PS levels than other PROS1 variants. In participants with reduced PS levels, the frequency of VTE was higher when a coding PROS1 variant was present. These data suggest that molecular analysis of the PROS1 gene may provide clinical value in the diagnostic work-up of PS deficiency, including the potential for improved risk prediction for development of VTE.

\section{Ethics}

All participants gave written informed consent. The study was approved by the Central Denmark Region Committees on Biomedical Research Ethics (\#1-10-72-333-14) and the Danish Data Protection Agency (\#1-16-02525-14). The study was conducted according to the Helsinki Declaration. 


\section{Funding}

This research did not receive any specific grant from funding agencies in the public, commercial, or not-forprofit sectors.

\section{Conflict of Interests}

The authors declare no conflict of interests related to the present study.

\section{Acknowledgments}

We are grateful to the Department of Clinical Biochemistry for continuous support to this project and to the laboratory staff for expert technical assistance.

We thank the participants and their families for their willingness to participate in this study.

\section{References}

1 Dahlbäck B. The protein C anticoagulant system: inherited defects as basis for venous thrombosis. Thromb Res 1995;77(01):1-43

2 Comp PC, Esmon CT. Recurrent venous thromboembolism in patients with a partial deficiency of protein S. N Engl J Med 1984;311(24):1525-1528

3 Comp PC, Nixon RR, Cooper MR, Esmon CT. Familial protein S deficiency is associated with recurrent thrombosis. J Clin Invest 1984;74(06):2082-2088

4 Khan S, Dickerman JD. Hereditary thrombophilia. Thromb J 2006; 4:15

5 Martinelli I, De Stefano V, Mannucci PM. Inherited risk factors for venous thromboembolism. Nat Rev Cardiol 2014;11(03):140-156

6 Mannucci PM, Franchini M. The real value of thrombophilia markers in identifying patients at high risk of venous thromboembolism. Expert Rev Hematol 2014;7(06):757-765

7 Maruyama K, Kokame K. Carrier frequencies of antithrombin, protein $\mathrm{C}$, and protein $\mathrm{S}$ deficiency variants estimated using a public database and expression experiments. Res Pract Thromb Haemost 2020;5(01):179-186

8 Makris M, Leach M, Beauchamp NJ, et al. Genetic analysis, phenotypic diagnosis, and risk of venous thrombosis in families with inherited deficiencies of protein S. Blood 2000;95(06): 1935-1941

9 Vossen CY, Conard J, Fontcuberta J, et al; The European Prospective Cohort on Thrombophilia (EPCOT) Risk of a first venous thrombotic event in carriers of a familial thrombophilic defect. J Thromb Haemost 2005;3(03):459-464

10 Vossen CY, Conard J, Fontcuberta J, et al. Familial thrombophilia and lifetime risk of venous thrombosis. J Thromb Haemost 2004;2 (09):1526-1532

11 Vossen CY, Walker ID, Svensson P, et al. Recurrence rate after a first venous thrombosis in patients with familial thrombophilia. Arterioscler Thromb Vasc Biol 2005;25(09):1992-1997

12 Di Minno MN, Ambrosino P, Ageno W, Rosendaal F, Di Minno G, Dentali F. Natural anticoagulants deficiency and the risk of venous thromboembolism: a meta-analysis of observational studies. Thromb Res 2015;135(05):923-932

13 Marlar RA, Gausman JN. Protein S abnormalities: a diagnostic nightmare. Am J Hematol 2011;86(05):418-421

14 Marlar RA, Gausman JN, Tsuda H, Rollins-Raval MA, Brinkman HJM. Recommendations for clinical laboratory testing for protein S deficiency: communication from the SSC committee plasma coagulation inhibitors of the ISTH. J Thromb Haemost 2021;19 (01):68-74

15 Caspers M, Pavlova A, Driesen J, et al. Deficiencies of antithrombin, protein $C$ and protein $S$ - practical experience in genetic analysis of a large patient cohort. Thromb Haemost 2012;108 (02):247-257

16 den Dunnen JT, Dalgleish R, Maglott DR, et al. HGVS Recommendations for the Description of Sequence Variants: 2016 Update. Hum Mutat 2016;37(06):564-569

17 Adzhubei IA, Schmidt S, Peshkin L, et al. A method and server for predicting damaging missense mutations. Nat Methods 2010;7 (04):248-249

18 Sim NL, Kumar P, Hu J, Henikoff S, Schneider G, Ng PC. SIFT web server: predicting effects of amino acid substitutions on proteins. Nucleic Acids Res 2012;40(Web Server issue):W452-7

19 Schwarz JM, Cooper DN, Schuelke M, Seelow D. MutationTaster2: mutation prediction for the deep-sequencing age. Nat Methods 2014;11(04):361-362

20 Reese MG, Eeckman FH, Kulp D, Haussler D. Improved splice site detection in Genie. J Comput Biol 1997;4(03):311-323

21 Brunak S, Engelbrecht J, Knudsen S. Prediction of human mRNA donor and acceptor sites from the DNA sequence. J Mol Biol 1991; 220(01):49-65

22 Reese MG. Application of a time-delay neural network to promoter annotation in the Drosophila melanogaster genome. Comput Chem 2001;26(01):51-56

23 Richards S, Aziz N, Bale S, et al; ACMG Laboratory Quality Assurance Committee. Standards and guidelines for the interpretation of sequence variants: a joint consensus recommendation of the American College of Medical Genetics and Genomics and the Association for Molecular Pathology. Genet Med 2015;17(05): 405-424

24 Li L, Wu X, Wu W, Ding Q Cai X, Wang X. Clinical manifestation and mutation spectrum of 53 unrelated pedigrees with protein $S$ deficiency in China. Thromb Haemost 2019;119(03):449-460

25 Alhenc-Gelas M, Canonico M, Morange PE, Emmerich JGeht Genetic Thrombophilia Group. Protein $S$ inherited qualitative deficiency: novel mutations and phenotypic influence. J Thromb Haemost 2010;8(12):2718-2726

26 Masica DL, Karchin R. Towards increasing the clinical relevance of in silico methods to predict pathogenic missense variants. PLOS Comput Biol 2016;12(05):e1004725

27 Pintao MC, Garcia AA, Borgel D, et al. Gross deletions/duplications in PROS1 are relatively common in point mutation-negative hereditary protein S deficiency. Hum Genet 2009;126(03): 449-456

28 Labrouche-Colomer S, Soukarieh O, Proust C, et al; GenMed Consortium. A novel rare c.-39C $>\mathrm{T}$ mutation in the PROS1 5'UTR causing PS deficiency by creating a new upstream translation initiation codon. Clin Sci (Lond) 2020;134(10): $1181-1190$

29 Halvorsen M, Lin Y, Sampson BA, et al. Whole exome sequencing reveals severe thrombophilia in acute unprovoked idiopathic fatal pulmonary embolism. EBioMedicine 2017;17:95-100

30 Ten Kate MK, Platteel M, Mulder R, et al. PROS1 analysis in 87 pedigrees with hereditary protein $\mathrm{S}$ deficiency demonstrates striking genotype-phenotype associations. Hum Mutat 2008;29 (07):939-947

31 Dykes AC, Walker ID, McMahon AD, Islam SI, Tait RC. A study of protein $S$ antigen levels in 3788 healthy volunteers: influence of age, sex and hormone use, and estimate for prevalence of deficiency state. Br J Haematol 2001;113(03):636-641

32 Gandrille S, Borgel D, Eschwege-Gufflet V, et al. Identification of 15 different candidate causal point mutations and three polymorphisms in 19 patients with protein $S$ deficiency using a scanning method for the analysis of the protein $\mathrm{S}$ active gene. Blood 1995;85(01):130-138

33 Downes K, Megy K, Duarte D, et al; NIHR BioResource. Diagnostic high-throughput sequencing of 2396 patients with bleeding, thrombotic, and platelet disorders. Blood 2019;134(23): 2082-2091 
34 Duebgen S, Kauke T, Marschall C, et al. Genotype and laboratory and clinical phenotypes of protein s deficiency. Am J Clin Pathol 2012;137(02):178-184

35 Amendola LM, Dorschner MO, Robertson PD, et al Actionable exomic incidental findings in 6503 participants: challenges of variant classification. Genome Res 2015;25(03): 305-315

36 Simmonds RE, Ireland H, Kunz G, Lane DAProtein S Study Group. Identification of 19 protein $S$ gene mutations in patients with phenotypic protein S deficiency and thrombosis. Blood 1996;88 (11):4195-4204
37 Wypasek E, Corral J, Alhenc-Gelas M, et al. Genetic characterization of antithrombin, protein $\mathrm{C}$, and protein $\mathrm{S}$ deficiencies in Polish patients. Pol Arch Intern Med 2017;127(7-8):512-523

38 Andersen BD, Bisgaard ML, Lind B, Philips M, Villoutreix B, Thorsen S. Characterization and structural impact of five novel PROS1 mutations in eleven protein S-deficient families. Thromb Haemost 2001;86(06):1392-1399

39 Beauchamp NJ, Dykes AC, Parikh N, Campbell Tait R, Daly ME. The prevalence of, and molecular defects underlying, inherited protein $\mathrm{S}$ deficiency in the general population. Br J Haematol 2004; 125(05):647-654 